\title{
Electron Bunch Acceleration by an Intense Laser Pulse with a Plasma Separator
}

\author{
Kei Sakai* Non-member \\ Kouichi Miyauchi* Non-member \\ Shuji Miyazaki* Non-member \\ Qing Kong** Non-member \\ Takashi Kikuchi* Non-member \\ Shigeo Kawata* Member
}

\begin{abstract}
We study an electron acceleration by using a focused $\mathrm{TEM}_{10}+\mathrm{TEM}_{01}$ mode laser pulse with a plasma separator in vacuum. By using the $\mathrm{TEM}_{10}+\mathrm{TEM}_{01}$ mode laser and the plasma separator, the electrons are accelerated strongly compared with that in the case of a $\mathrm{TEM}_{00}$ mode laser, and the divergence of the electron bunch accelerated is suppressed in the transverse direction by the transverse ponderomotive force of the laser. In both the $\mathrm{TEM}_{00}$ mode laser and the $\mathrm{TEM}_{10}+\mathrm{TEM}_{01}$ mode laser, the electrons are decelerated by the laser at the deceleration phase of the laser pulse tail. In order to suppress the energy loss, we set an overdense plasma separator before the electrons are decelerated by the laser tail. Only the laser pulse is reflected by the plasma separator, and the high energy electrons pass through the plasma separator without the energy loss at the tail of laser pulse.
\end{abstract}

Keywords: electron accelerator, laser accelerator, plasma separator, intense laser

\section{Introduction}

In resent years, intense short pulse lasers became available by remarkable progresses in a laser technology ${ }^{(1)(2)}$, and the laser intensity reaches as high as $\sim 10^{25}\left[\mathrm{~W} / \mathrm{m}^{2}\right]$. The intense laser field provides a strong field gradient more than $10^{12}[\mathrm{~V} / \mathrm{m}]$, which may provide new particle acceleration schemes ${ }^{(3)-(13)}$, including an electron acceleration in vacuum. In this paper we propose an electron acceleration mechanism by using an intense short pulse laser of $\mathrm{TEM}_{10}+\mathrm{TEM}_{01}{ }^{(14)-(16)}$ mode and a plasma separator. In general, a $\mathrm{TEM}_{00}$ mode laser is widely used by a particle acceleration. The $\mathrm{TEM}_{00}$ mode laser has a peak of a field strength in the central axis. Therefore, the electrons are accelerated by the longitudinal ponderomotive force at the head of the laser pulse, and are scattered by the transverse pondermotive force in the transverse direction. This is one of the serious problems in the electron acceleration by the laser. In order to suppress the transverse electron scattering, we employ the $\mathrm{TEM}_{10}+\mathrm{TEM}_{01}$ mode laser. The $\mathrm{TEM}_{10}+\mathrm{TEM}_{01}$ mode laser has two intensity peaks in the transverse direction, and the electrons are confined by the transverse ponderomotive force near the central axis. The longitudinal electric field of the $\mathrm{TEM}_{10}+\mathrm{TEM}_{01}$ mode laser

\footnotetext{
* Department of Electrical and Electronic Engineering, Utsunomiya University

Yohtoh 7-1-2, Utsunomiya 321-8585

** Institute of Modern Physics, Fudan University

220, Handan road, Yangpu district, Shanghai, China
}

becomes strong at the central axis. Although the acceleration of the $\mathrm{TEM}_{00}$ mode laser is driven by the longitudinal ponderomotive force, the $\mathrm{TEM}_{10}+\mathrm{TEM}_{01}$ mode laser accelerates electrons by the longitudinal electric field. However, if the laser intensity is enough high or the velocity of the electrons injected is nearly $c$, the electrons keep the position in the acceleration phase of one cycle of laser near the laser focal point for a relatively longer time. Here $c$ is the speed of light in vacuum. Therefore the electrons are accelerated well by the longitudinal electric field and confined by the transverse ponderomotive force.

In the $\mathrm{TEM}_{00}$ mode laser, the electrons are accelerated by the longitudinal ponderomotive force in the front of laser pulse. However, at the tail of the laser pulse, the electrons are decelerated and lose their energy. Even if the $\mathrm{TEM}_{10}+\mathrm{TEM}_{01}$ mode laser, such the energy loss appears. In order to obtain a net acceleration energy, we need to overcome the Lawson-Woodward theorem ${ }^{(17)}$ by introducing some ideas, for example, setting a boundary to separate the accelerated electrons from the laser pulse, using the inverse processes and so on.

In this paper we also propose a plasma separator to extract electrons from the focused $\mathrm{TEM}_{10}+\mathrm{TEM}_{01}$ mode laser. At first, the electrons are accelerated by the laser field, and then the laser pulse and the electrons accelerated hit the overdense plasma separator. The laser is reflected and the accelerated electrons pass through the thin plasma separator. As a result, the accelerated electrons are separated from the laser by the plasma 
separator and the electrons obtain the net energy from the laser ${ }^{(18)(19)}$

In Section 2, we present a simulation model and parameters. In Section 3, we discribe the simulation results of the electrons acceleration and separation from the $\mathrm{TEM}_{10}+\mathrm{TEM}_{01}$ mode laser by using the plasma separator. The conclusions are shown in Section 4.

\section{Simulation Model}

Figure 1 shows a schematic view of our plasmaseparator simulation model in 2.5-dimensional particlein-cell (PIC) ${ }^{(20)}$ simulations. In the case with the $\mathrm{TEM}_{10}+\mathrm{TEM}_{01}$ mode laser pulse, the 2.5-dimensional $\left((z, r),\left(v_{z}, v_{r}, v_{\theta}\right)\right)$ cylindrical coordinate is used, and the 2.5-dimensional $\left((x, y),\left(v_{x}, v_{y}, v_{z}\right)\right)$ Cartesian coordinate is employed for the calculations in the case of $\mathrm{TEM}_{00}$ mode laser. In this paper we adopt the focused Gauss pulse, and the laser is injected at the left boundary of the simulation box. The transverse and longitudinal electric fields of the $\mathrm{TEM}_{10}+\mathrm{TEM}_{01}$ mode laser pulse given at the left boundary are written by

$$
\begin{aligned}
E_{r}= & 2 \sqrt{2} E_{0} r \frac{w_{0}}{w^{2}} \exp \left[-\frac{r^{2}}{w^{2}}-\frac{\left(z_{0}+c t\right)^{2}}{(\tau / 2)^{2}}\right] \\
& \times \sin \left(-k z_{0}-\omega t-2 \phi+\frac{k r^{2}}{2 R}\right), \cdots \\
E_{z}= & \frac{i}{k} \frac{\partial E_{r}}{\partial r}, \ldots \ldots \ldots \ldots \ldots \ldots \ldots
\end{aligned}
$$

where

$$
\begin{aligned}
& w=w_{0} \sqrt{1+\frac{z_{0}^{2}}{z_{R}^{2}}}, \ldots \ldots \ldots \ldots \ldots \ldots \ldots \ldots \\
& R=-z_{0}\left[1+\frac{z_{R}^{2}}{z_{0}^{2}}\right] \\
& \phi=\tan ^{-1}\left(\frac{-z_{0}}{z_{R}}\right) .
\end{aligned}
$$

Here $z_{R}=\pi w_{0}^{2} / \lambda$ is the Rayleigh length of laser, $E_{0}$ is the amplitude of the electric field, $w_{0}$ is the minimum laser spot size, $z_{0}$ is the focal point of the laser, $\tau$ is the pulse length of the laser, $c$ is the light velocity, $k$ is the wave number of the laser, $\omega$ is the angular frequency of the laser, and $\lambda$ is the laser wave length of $1.053[\mu \mathrm{m}]$ in this paper. In the case with the $\mathrm{TEM}_{00}$ mode laser pulse, the transverse and the longitudinal component of the electric fields given at the left boundary are given by

$$
\begin{aligned}
E_{y}= & E_{0} \frac{w_{0}}{w} \exp \left[-\frac{y^{2}}{w^{2}}-\frac{\left(x_{0}+c t\right)^{2}}{(\tau / 2)^{2}}\right] \\
& \times \sin \left(-k x_{0}-\omega t-\phi+\frac{k y^{2}}{2 R}\right), \\
E_{x}= & \frac{i}{k} \frac{\partial E_{y}}{\partial y} . \cdots \ldots \ldots \ldots \ldots \ldots
\end{aligned}
$$

Here $x_{0}$ is the focal point with the case of the $\mathrm{TEM}_{00}$ mode laser. In our simulations, the laser intensity is $I=1.8 \times 10^{23}\left[\mathrm{~W} / \mathrm{m}^{2}\right]\left(a_{0} \sim 2.7, E_{0}=1.16 \times 10^{13}[\mathrm{~V} / \mathrm{m}]\right)$, the focal point of the laser $z_{0}=x_{0}=15 \lambda$, the minimum laser spot size $w_{0}=3 \lambda$, and the pulse length $\tau$

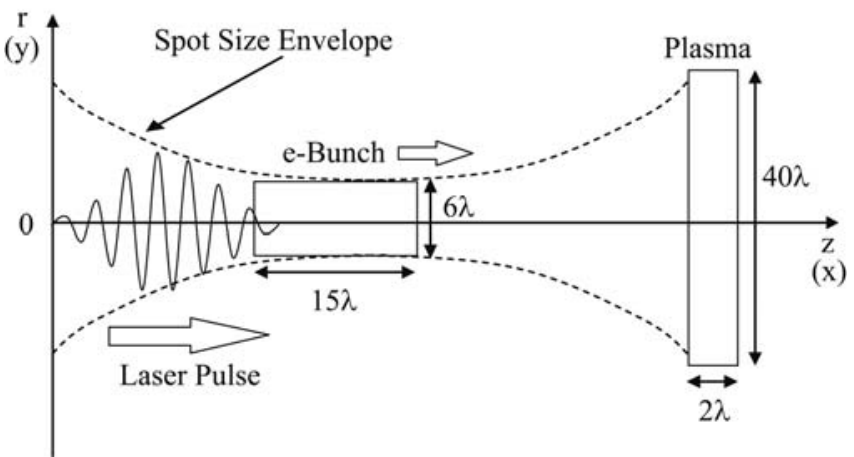

Fig. 1. Simulation model; The laser is injected at the left boundary at $t=0$. We set that the laser intensity peak and the electron bunch center coincide with each other at the focal point $(z, r)=(x, y)=$ $(15 \lambda, 0)$

$=15 \lambda$. Here $a_{0}$ is the dimensionless amplitude parameter of the laser. These equations are used at the left boundary of the computation area, and then the laser propagation and the electromagnetic field are solved by the Maxwell equation. The relativistic equation of motion $\mathrm{d} \boldsymbol{P} / \mathrm{d} t=q(\boldsymbol{E}+\boldsymbol{V} \times \boldsymbol{B})$ is solved, where $\boldsymbol{P}$ is the particle momentum and $q$ the particle charge. The initial electron bunch moves to the longitudinal direction at a speed of $0.1 c$ and the initial electron bunch density is $1.0 \times 10^{20}\left[\mathrm{~m}^{-3}\right]$. In order to coincide the center of the electron bunch and the laser intensity peak at the focal point, the electron bunch is located at $5 \lambda \leq z \leq$ $20 \lambda$, and its transverse size is $0 \leq r \leq 3 \lambda$ at the initial time. The separator plasma is a hydrogen plasma with a density of $3 \mathrm{n}_{c}$, where $\mathrm{n}_{c}$ is the critical density; the standard separator plasma is located at $35 \leq z \leq$ $37 \lambda$ and its transverse size is $0 \leq r \leq 20 \lambda$ so that the accelerated electrons do not meet the deceleration phase at the laser tail. The initial plasma separator position is varied for a parameter survey. The electrons and the separator plasma are in the Maxwell distributions with the electron bunch temperature of $T_{e}=1[\mathrm{eV}]$ and the plasma separator temperature $T_{p}=100[\mathrm{eV}]$ at the initial time, respectively. The interaction between the plasma separator and the laser is self-consistently solved by the PIC simulation method ${ }^{(20)}$.

\section{Simulation Results}

3.1 Electron Acceleration Process Figures 2a), b), c) and d) show the maps of electron cloud and energy $\gamma$ maps versus $x$ in the case with the $\mathrm{TEM}_{00}$ mode laser pulse at the time of $t=169[\mathrm{fs}]$ and $t=253[\mathrm{fs}]$. The electrons are accelerated longitudinally by the ponderomotive force of the laser pulse near the central axis, and the electrons accelerated are pushed toward the head of the electron bunch. The electrons accelerted enter the laser deceleration regions because of the different phase velocity between the laser and electrons. Consequently the electrons are decelerated by the ponderomotive force and are scattered in the transverse direction during the laser field expansion as shown in Figs. 2a), b), c), and d). Figures 2e) and g) present the snapshots of 

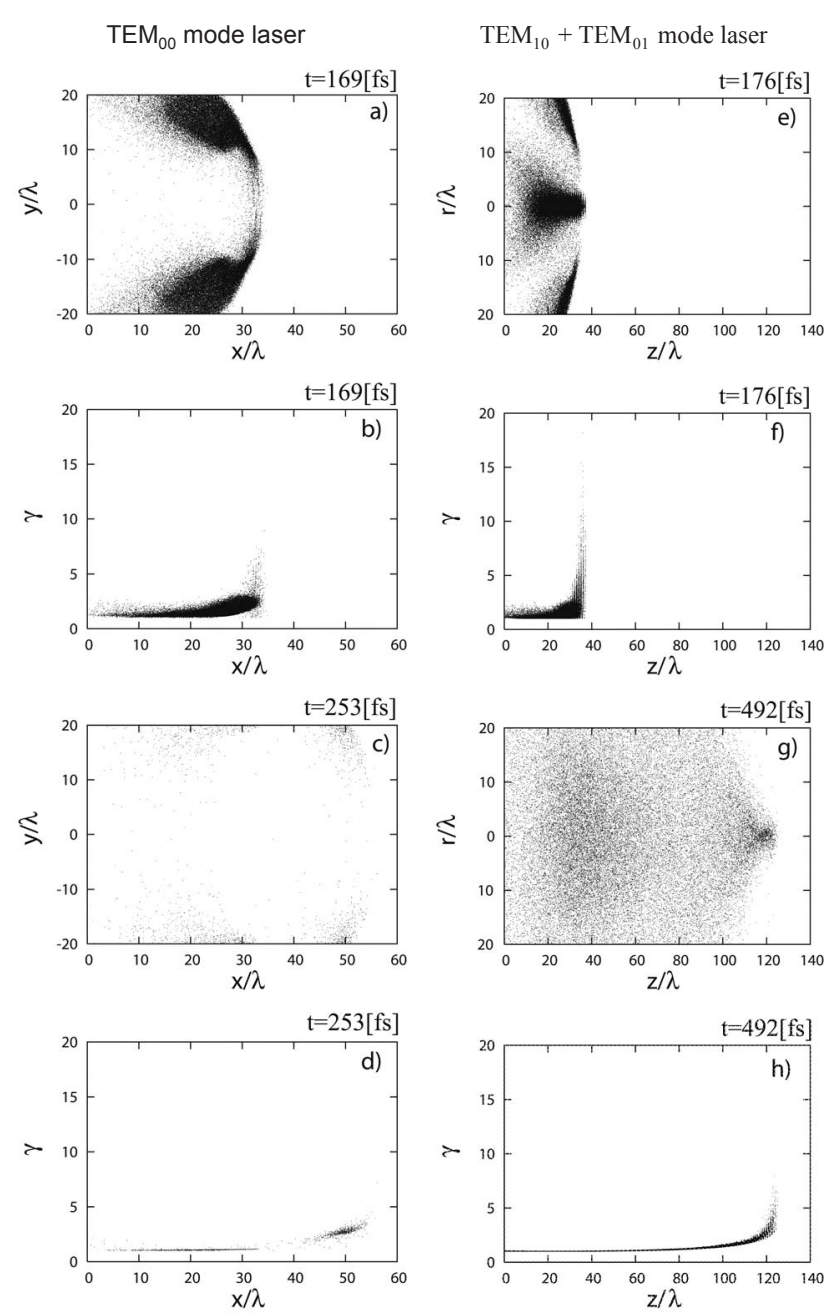

Fig. 2. The electron distributions in $x-y$ and the electron energy distributions in the cases of the $\mathrm{TEM}_{00}$ mode laser and the $\mathrm{TEM}_{10}+\mathrm{TEM}_{01}$ mode laser; In these results the plasma separator is not employed. The left side figures show the results in the case of the $\mathrm{TEM}_{00}$ mode laser pulse. The right side figures show the results in the case of the $\mathrm{TEM}_{10}+\mathrm{TEM}_{01}$ mode laser pulse. a) and c) show the electrons maps in the $x-y$ plane at the time of $t=169[\mathrm{fs}]$ and $t=253[\mathrm{fs}]$ in the case of the $\mathrm{TEM}_{00}$ mode laser pulse respectively, and b) and d) present the electrons energy distributions in the longitudinal direction at $t=169$ [fs], and $t=253[\mathrm{fs}$ ] respectively. The electron distributions in the $z-r$ plane and the energy distributions are shown in e), f), g), and h) at $t=176[\mathrm{fs}]$, and $t=492[\mathrm{fs}]$ in the case of the $\mathrm{TEM}_{10}+\mathrm{TEM}_{01}$ mode laser

the electrons in $z-r$ at the $t=176[\mathrm{fs}]$, and $t=492[\mathrm{fs}]$, and Figs. 2f) and h) show the electron energy $\gamma$ distributions as a function of $z$ in the case with the $\mathrm{TEM}_{10}+\mathrm{TEM}_{01}$ mode laser pulse at $t=176[\mathrm{fs}]$, and $t=492[\mathrm{fs}]$. By using the $\mathrm{TEM}_{10}+\mathrm{TEM}_{01}$ mode laser pulse, the electrons are accelerated near the focal point, and the transverse ponderomotive force confines the electrons near the laser axis as shown in Figs. 2e) and g). From Figs. 2d) and $\mathrm{h}$ ) one can see the difference of the electron energy $\gamma$ for the $\mathrm{TEM}_{00}$ mode laser (The average $\gamma$ is 4.1.) and the $\mathrm{TEM}_{10}+\mathrm{TEM}_{01}$ mode laser (The average $\gamma$ is 7.9.).

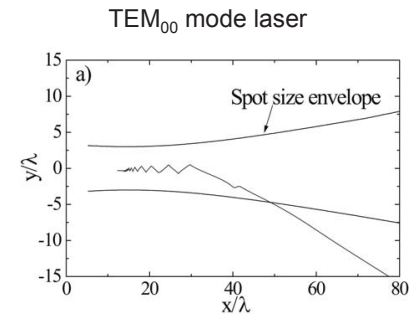

$\mathrm{TEM}_{10}+\mathrm{TEM}_{01}$ mode laser
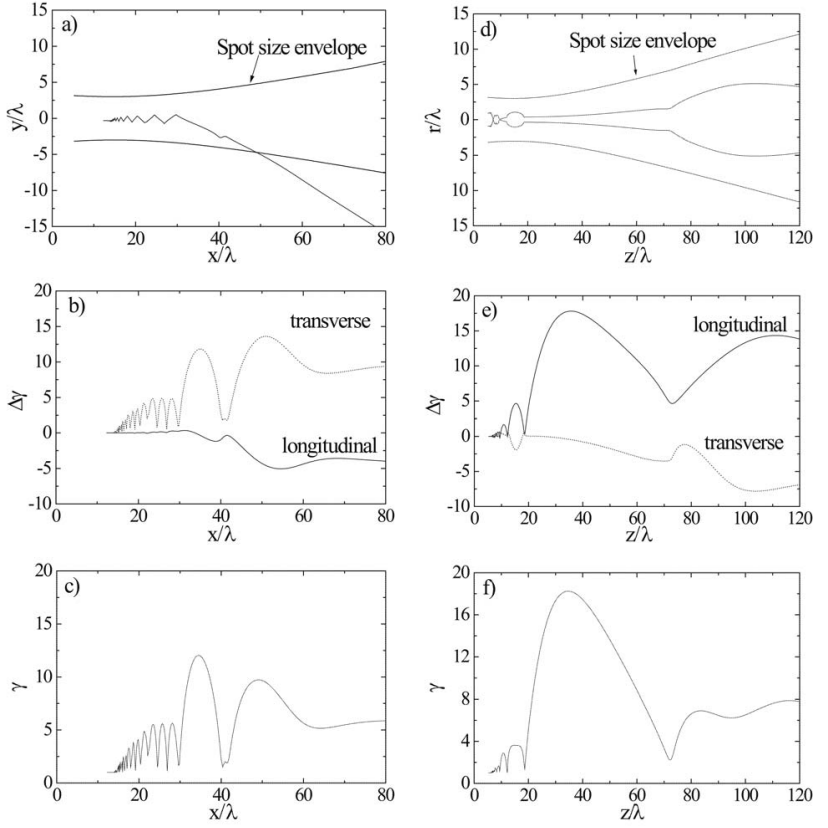

Fig. 3. The accelerated electrons trajectories in the $x-y$ and the $z-r$ planes; The energy exchange and electron energy trajectories in the longitudinal direction. The left side figures show the results in the case with the $\mathrm{TEM}_{00}$ mode laser pulse and the right side figures present the results in the case with the $\mathrm{TEM}_{10}+\mathrm{TEM}_{01}$ mode laser pulse. Figures a) and d) show the electrons trajectories. Figures b) and e) show the energy exchange $\Delta \gamma$ from the longitudinal (a solid line) and the transverse (a dotted line) electric fields. c) and f) present the electron energy history

In the $\mathrm{TEM}_{10}+\mathrm{TEM}_{01}$ mode laser, the longitudinal electric field becomes high in the central axis. In the parameters we employed the laser intensity is high $(I=$ $\left.1.8 \times 10^{23}\left[\mathrm{~W} / \mathrm{m}^{2}\right]\right)$ and the minimal spot size is small $\left(w_{0}=3.0 \lambda\right)$, the electrons are accelerated up to the relativistic energies by the longitudinal electric field(see Fig. 3e)). The maximum longitudinal electric field $E_{z}$ is $3.18 \times 10^{11}[\mathrm{~V} / \mathrm{m}]$ at the focal point.

Figure 3 presents typical electron behaviors and in Fig.3 an electron, which has the largest $\gamma$ at the peak energy, is selected in each laser mode. Figure 3a) shows the trajectory of the well-accelerated electron in the case with the $\mathrm{TEM}_{00}$ mode laser pulse by the electron bunch calculation. Figure $3 \mathrm{~b}$ ) shows the energy exchange $\Delta \gamma$ from the longitudinal electric field (a solid line) and the transverse electric field (a dotted line) in the case with the $\mathrm{TEM}_{00}$ mode laser pulse. In Fig. 3b) the electron is accelerated and obtains energy by the transverse electric field. Figure 3c) shows the energy of the electron with the $\mathrm{TEM}_{00}$ mode laser pulse. The electron receives energy of about $\gamma=12.0$, that is, $6.1[\mathrm{MeV}]$, but the electron is decelerated by the laser pulse as shown in Fig. 3c). Figure 3d) shows the trajectory of the wellaccelerated electron in the case by using the $\mathrm{TEM}_{10}+$ $\mathrm{TEM}_{01}$ mode laser pulse by the electron bunch calculation. In Fig. 3d) the electron is accelerated at the 

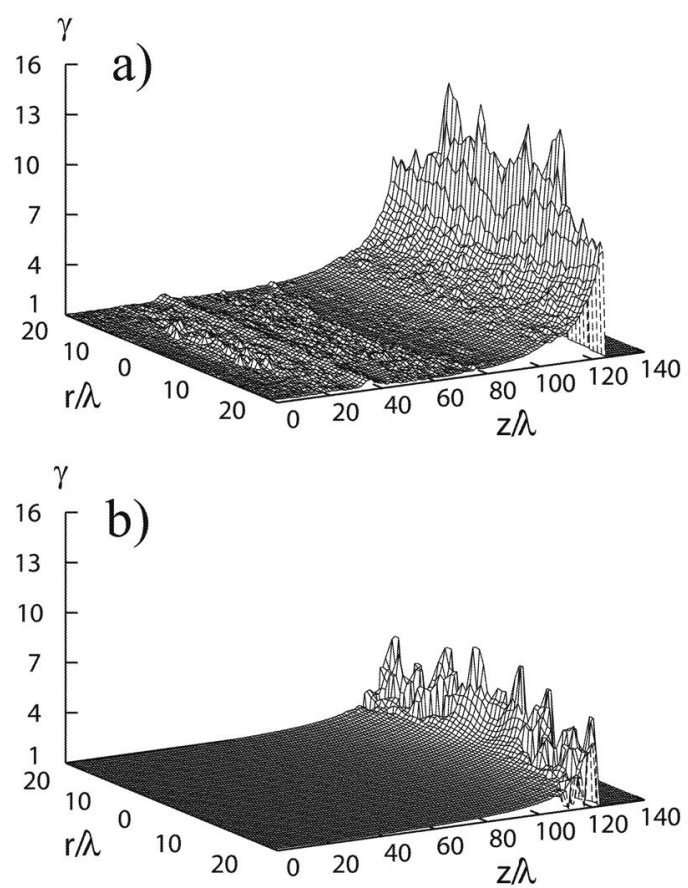

Fig. 4. The electron energy distributions in the $z-r$ plane at the $t=492[\mathrm{fs}]$; a) The case with the plasma separator and b) the case without the plasma separator

center to the direction of an axis of the laser pulse. Figure 3e) shows the energy exchange $\Delta \gamma$ from the longitudinal electric field (a solid line) and the transverse electric field (a dotted line) in the case with the $\mathrm{TEM}_{10}$ $+\mathrm{TEM}_{01}$ mode laser pulse. In Fig. 3e) the electron is accelerated and gets energy mainly by the longitudinal electric field. Figure 3f) shows the energy of the electron with the $\mathrm{TEM}_{10}+\mathrm{TEM}_{01}$ mode laser pulse. In Fig. 3f) the electron receives the maximum energy of about $\gamma=$ 18.3, that is, $9.4[\mathrm{MeV}]$, but the electron is decelerated by the laser tail.

3.2 Electron Bunch Acceleration by the TEM $_{10}$ + TEM $_{01}$ Mode Laser Pulse with Plasma Separator Figure 4 shows maps of electrons energy for the cases with the plasma separator (see Fig. 4a)) and without the plasma (see Fig. 4b)) at 492 [fs]. In Fig. 4 the plasma electron energy is not presented. The electron energy in the case with the plasma separator is higher than that in the case without the separator. In the case with the separator, only the laser is reflected by the plasma separator and the electrons accelerated pass through the plasma separator without the deceleration at the laser tail. Therefore the electron energy is higher than that in the case without the plasma separator.

Figure 5 shows the trajectories of well-accelerated electrons energy after passing through the separator by using the $\mathrm{TEM}_{10}+\mathrm{TEM}_{01}$ mode laser pulse for the cases with (a solid line) and without (a dotted line) the plasma separator in a) the $z-r$ plane and in b) the $z-\gamma$ space. In both the cases the electrons are accelerated by the longitudinal electric field and are confined by the transverse ponderomotive force. However in the
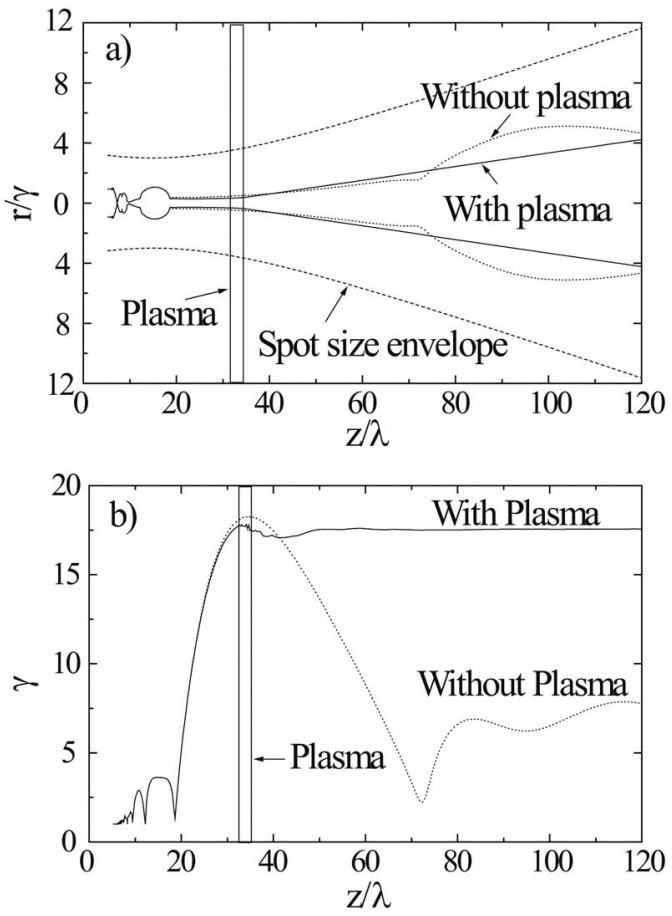

Fig. 5. The well-accelerated electron trajectories and the energy histories with the plasma separator (a solid line) and without the separator (a dotted line). Figure a) shows the electron trajectories in the $z-r$ space. Figure b) presents the electron energy histories versus the electron position of $z$

case without the plasma separator the electrons enter to the deceleration phase of the laser and loose their energy. On the other hand, the electrons keep their energy after the interaction with the plasma separator. In the case without the plasma separator, the maximum electron energy about $\gamma=7.9$, that is $4.03[\mathrm{MeV}]$ as shown in Fig. 5b). On the other hand, the electron final energy in the case with the plasma separator, $\gamma=17.5$, that is $8.94[\mathrm{MeV}]$. From Fig. 5b) one can see the electron is slightly decelerated by the reflected laser field, before the electron passes the plasma separator. In addition, at the right side edge of the plasma separator, plasma electrons, kicked by the laser, induce an ambipolar electric field ${ }^{(21)}$, which reachs about $10^{11}[\mathrm{~V} / \mathrm{m}]$ at its maximum. However, the ambipolar field is localized spatially in a short length of several $\lambda \sim 10 \lambda$ and has a positive potential peak and a negative potential peak to ensure the cherge neutrality. Therefore in a short distance the beam electrons experience a slight energy change just after passing through the plasma separator, and the beam electrons neither gain nor loose a large energy at last. As shown in Fig. 5b) this effect is minor, because the beam electron accelerated has a sufficient high energy compared to the ambipolar potential energy.

Figures 6a) and b) show the results of the parameter study. Figure 6a) presents a relation between the plasma separator position and the electron beam average energy, and Fig. 6b) shows the relation between the separator position and the emittances. The results shown in Figs. $6 \mathrm{a}$ ) and b) present that there is the optimal position 

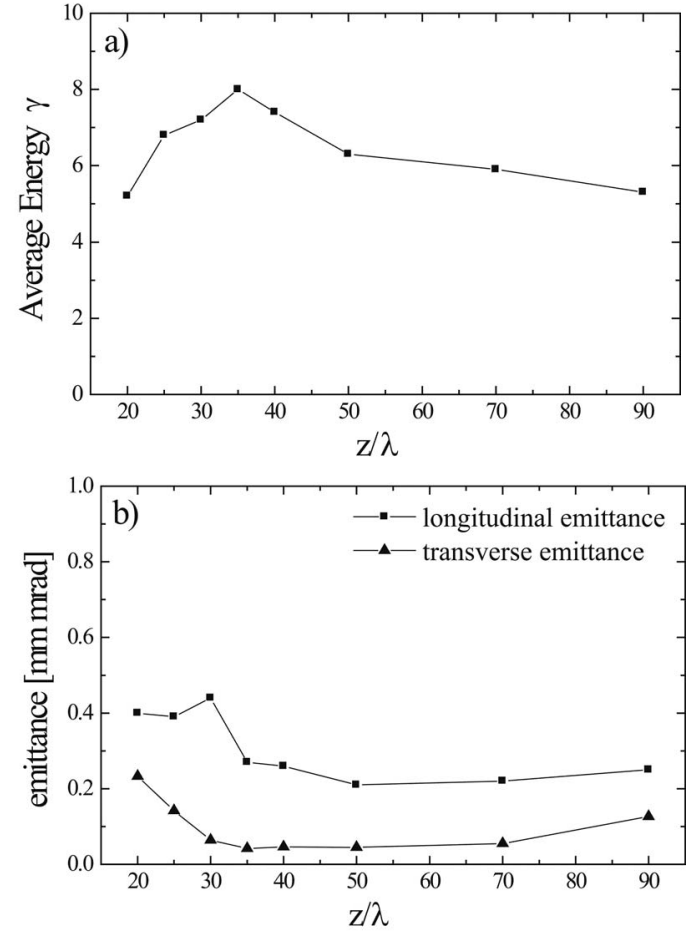

Fig. 6. Parameter study results; a) The separated beam electron average energy vs. the left side position of the plasma separator, b) The transverse and the longitudinal emittances of the separated beam electron vs. the left side position of the separator

of the plasma separator. When the plasma separator is put left in $z$ from the optimal position, the electrons are not yet accelerated sufficiently and therefore the electron average energy becomes lower. The insufficient acceleration also leads larger emittances. When the plasma separator is put right in $z$, the electrons experience the laser deceleration phase. At this deceleration phase the electron energy is already large and the deceleration is slow, compared with the acceleration (see the dotted line in Fig. 5b), in which one can see that the acceleration phase has a steep gradient of $\gamma$ compared with that in the deceleration phase). Especially the emittance expression used are proportional to the inverse of the average longitudinal momentum ${ }^{(22)}$. Therefore the decrease in $\gamma$ and the increase in the emittances can be explained by the difference between the acceleration length and the deceleration length. Figure 6 presents that the electron beam average energy and the longitudinal and the transverse emittances are relatively insensitive to the plasma separator position in practical.

The thickness of the plasma separator should be thicker than the skin depth, and at the same time should be sufficiently thin so that the electrons do not meet a significant influence from the plasma separator itself: the collision and instability effects should be suppressed sufficiently. In our present parameter range, $d_{e} \ll l$. Here $d_{e}$ is the skin depth and $l$ is the plasma separator thickness. Therefore the opaque condition is fulfilled in this paper. The maximum growth rate of the twostream instability between the beam electrons and the separator electrons is given by ${ }^{(23)}$

$$
\delta_{\max }=\omega_{p} \sqrt{\frac{\pi}{2}} \frac{n_{b}}{n_{p}} \frac{V_{b}^{2}}{u_{b}^{2}} \exp \left(-\frac{1}{2}\right),
$$

under the conditions of $\omega_{p}^{2} /\left(k u_{p}\right)^{2} \gg 1, n_{b} / n_{p} \ll 1$ and $V_{b}^{2} \gg u_{p}^{2}$. Here $\omega_{p}$ is the beam electron plasma frequency, $n_{b}$ is the beam electron density, $n_{p}$ is the plasma separator electron density, $V_{b}$ is the beam speed, $u_{b}$ is the beam thermal speed, $u_{p}$ is the plasma separator electron thermal speed and $k$ is the wave number. In our cases $\omega_{p}=5.19 \times 10^{14}[1 / \mathrm{s}], n_{b}=1.40 \times 10^{20}\left[1 / \mathrm{m}^{3}\right]$, $n_{p}=3.02 \times 10^{27}\left[1 / \mathrm{m}^{3}\right], \quad V_{b}=2.97 \times 10^{8} \quad[\mathrm{~m} / \mathrm{s}] \quad$ and $u_{b}=$ $1.41 \times 10^{5}[\mathrm{~m} / \mathrm{s}]$. The plasma separator passage time $\tau_{\text {ele }}$ of electrons is about $\tau_{\text {ele }}=7.08[\mathrm{fs}]$. The two-stream instability maximum growth rate is $\delta_{\max }=5.81 \times 10^{11}[1 / \mathrm{s}]$. This means the two stream instability is not so dengerous. The maximum growth rate of the filamentation instability between the beam electrons and the separator electrons is given by ${ }^{(24)}$

$$
\delta_{\max } \simeq \frac{\nu}{3} \beta, \cdots \ldots \ldots \ldots \ldots \ldots \ldots \ldots \ldots \ldots \ldots
$$

at

$$
k=\left(\frac{2}{9} \frac{\omega_{b}^{2}}{\nu^{2}}\right)^{1 / 4} \frac{\omega_{p}}{c}
$$

where $\beta=\omega_{b}^{2} / \omega_{p}^{2}+k^{2} c^{2} / \omega_{p}^{2}, \quad \nu$ is collision frequency among the plasma separator electrons and $\omega_{b}$ is the beam electron plasma frequency. Equation (9) is derived under the conditions of $|\zeta|>1,|\eta|>1$ and $\nu>\delta$, where $\xi=(\omega+i \nu) /\left(k u_{p}\right)$ and $\eta=\omega /\left(k u_{p}\right)$, and in this paper these conditions are fulfilled. Here $\omega$ is the complex wave frequency. In our case $\nu=6.80 \times 10^{13}[1 / \mathrm{s}]$, and $\omega_{b}=1.96 \times 10^{11}[1 / \mathrm{s}]$. Then the maximum growth rate is $\delta_{\max }=1.40 \times 10^{11}[1 / \mathrm{s}]$, and $\delta_{\max } \tau_{\text {ele }}=9.9 \times 10^{-4} \ll 1.0$. If the plasma separator is not too thick in its width, it serves a stable plasma separator. The electron energy loss by the collisions is estimated by ${ }^{(25)}$

$$
\left\langle\frac{d \epsilon}{d t}\right\rangle \simeq-\frac{e^{4} \ln \Lambda}{4 \pi \epsilon_{0}^{2}}\left(\frac{n_{p}}{m_{e}}\right) \frac{1}{V_{b}} \ldots \ldots \ldots \ldots \ldots
$$

Here $d \epsilon / d t$ is the energy loss of the accelerated electron by the collisions with the separator electrons and $e$ is the electron charge. $\ln \Lambda$ is the Coulomb logarithm, in our case $\ln \Lambda=3.88$. $\epsilon_{0}$ is the permittivity in vacuum and $m_{e}$ is the electron mass. The collision energy loss is about $1.3[\mathrm{eV}]$ in our parameter set. Therefore the electron energy loss by the collision is negligible.

\section{Conclusions}

In this paper we studied the electron acceleration by using the $\mathrm{TEM}_{10}+\mathrm{TEM}_{01}$ mode laser pulse with the plasma separator. The simulation results present that in the case with the $\mathrm{TEM}_{10}+\mathrm{TEM}_{01}$ mode laser pulse, the accelerated electrons energy is high compared with 
that in the case of the $\mathrm{TEM}_{00}$ mode laser pulse. The electrons accelerated by the $\mathrm{TEM}_{10}+\mathrm{TEM}_{01}$ mode laser pulse received energy from the longitudinal electric field. However the accelerated electrons lose their energy at the tail of the laser pulse. By setting the plasma separator before the electrons are decelerated, the electrons can obtain the net energy. In our study the influences of the plasma position are also investigated. The electron beam average energy and the emittance are relatively insensitive to the plasma separator position in practical.

\section{Acknowledgment}

This work was partly supported by the JSPS (Japan Society for the Promotion of Science) and MEXT (Ministry of Education, Culture, Sports, Science and Technology). We would like to present our thanks to Prof. K. Mima, Prof. K. Tachibana, Prof. S. Kurokawa, Prof. K. Nakajima, Prof. J. Limpouch, Prof. N. Yugami and Prof. S. V. Bulanov for their fruitful discussions on this subject.

(Manuscript received June 29, 2004,

revised Sep. 10, 2004)

\section{References}

(1) D. Strickland and G. A. Mourou: "Compression of amplified chirped optical pulses," Opt. Commun., Vol.56, pp.219-221 (1985)

(2) G. A. Mourou, C. P. J. Barty, and M. D. Perry: "UltrahighIntensity Lasers: Physics of the Extreme on a Tabletop," Phys. Today, Vol.51, pp.22-28 (1998)

( 3 ) T. Tajima and J. M. Dawson: "Laser Electron Accelerator," Phys. Rev. Lett., Vol.43, No.4, pp.267-270 (1979)

(4) M. O. Scully and M. S. Zubairy: "Simple laser accelerator: Optics and particle dynamics," Phys. Rev. A, Vol.44, No.4, pp.2656-2663 (1991)

(5) S. Kawata, T. Maruyama, H. Watanabe, and I. Takahashi: "Inverse-Bremsstrahlung Electron Acceleration," Phy. Rev. Lett., Vol.66, No.16, pp.2072-2075 (1991)

(6) L. C. Steinhauer and W. D. Kimurai: "A new approach for laser particle acceleration in vacuum," J. Appl. Phys., Vol.72, No.8, pp.3237-3245 (1992)

(7) E. Esarey, P. Sprangle, and J. Krall: "Laser acceleration of electrons in vacuum," Phys. Rev. E, Vol. 52, No. 5, pp. 5443 (1995)

(8) S. V. Bulanov, V. A. Vshivkov, G. I. Dudnikova, N. M. Naumova, F. Pegoraro, and I. V. Pogorelsky: "Laser Acceleration of Charged Particles in Inhomogeneous Plasmas. I," Plasma Phys. Rep., Vol.23, No.4, pp.259-269 (1997)

(9) G. Malka, E. Lefebvre, and J. L. Miquel: "Experimental Observation of Electrons Accelerated in Vacuum to Relativistic Energies by a High-Intensity Laser," Phys. Rev. Lett., Vol.78, No.17, pp.3314-3317 (1997)

(10) Q. Kong, Y. K. Ho, J. X. Wang, P. X. Wang, L. Feng, and Z. S. Yuan: "Conditions for electron capture by an ultraintense stationary laser beam," Phys. Rev. E, Vol.61, No.2, pp.1981$1984(2000)$

(11) S. C. Wilks, A. B. Langdon, T. E. Cowan, M. Roth, M. Singh, S. Hatchett, M. H. Key, D. Pennington, A. MacKinnon, and R. A. Snavely: "Energetic proton generation in ultra-intense laser-solid interactions," Phys. Plasmas, Vol.8, No.2, pp.542549 (2001)

(12) S. V. Bulanov, T. Zh. Esirkepov, V. S. Khoroshkov, A. V. Kuznetsov, and F. Pegoraro: "Oncological hadrontherapy with laser ion accelerators," Phys. Lett. A, Vol.299, No.2-3, pp.240-247 (2002)
(13) S. Miyazaki, Q. Kong, S. Kawata, and J. Limpouch: "Micro electron bunch generation by intense short pulse laser," $J$. Phys. D, Vol.36, pp.1-5 (2003)

(14) G. V. Stupakov and M. S. Zolotorev: "Ponderomotive Laser Acceleration and Focusing in Vacuum for Generation of Attosecond Electron Bunches," Phys. Rev. Lett., Vol.86, No.23, pp.5274-5277 (2001)

(15) Q. Kong, S. Miyazaki, S. Kawata, K. Miyauchi, K. Nakajima, S. Masuda, N. Miyanaga, and Y. K. Ho: "Electron bunch acceleration and trapping by the ponderomotive force of an intense short-pulse laser," Phys. Plasmas, Vol.10, No.12, pp.4605-4678 (2003)

(16) Q. Kong, S. Miyazaki, S. Kawata, K. Miyauchi, K. Sakai, Y. K. Ho, K. Nakajima, N. Miyanaga, J. Limpouch, and A. A. Andreev: "Electron bunch trapping and compression by an intense focused pulse laser," Phys. Rev. E, Vol.69, 056502 (2004)

(17) Y. I. Salamin and F. H. M. Faisal: "Harmonic generation by superintense light scattering from relativistic electrons," Phys. Rev. A, Vol.54, No.5, pp.4383-4395 (1996)

(18) W. Yu, V. Bychenko, Y. Sentoku, M. Y. Yu, Z. M. Sheng, and K. Mima: "Electron Acceleration by a Short Relativistic Laser Pulse at the Front of Solid Targets," Phys. Rev. Lett., Vol.85, No.3, pp.570-573 (2000)

(19) V. Vshivkov, N. Naumova, F. Pegoraro, and S. V. Bulanov: "Nonlinear electrodynamics of the interaction of ultra-intense laser pulse with a thin foil," Nuclear Instruments \& Methods in Physics Research A, Vol.410, pp.493-498 (1998)

(20) A. B. Langdon and B. F. Lasinski: "Electromagnetic and Relativistic Plasma Simulation Models," Methods Comput. Phys., Vol.16, pp.327-364 (1976)

(21) T. Nakamura and S. Kawata: "Origin of protons accelerated by an intense laser and the dependence of their energy on the plasma density," Phys. Rev. E, Vol.67, 026403 (2003)

(22) M. Reiser: Theory and Design of Charged Particle Beams, pp.393-395, John Wiley \& Sons, New York (1994)

(23) M. N. Rosenbluth, R. Z. Sagdeev, A. A. Galeev, and R. N. Sudan: Handbook of Plasma Physics Volume 1 Basic Plasma Physics I, pp.540-543, North-Holland, Amsterdam, Netherlands (1983)

(24) T. Okada and K. Niu: "Effect of collisions on the relativistic electromagnetic instability," J. Plasma Phys., Vol.24, pp.483488 (1980)

(25) K. Miyamoto: Plasma Physics for Nuclear Fusion, p. 78, MIT Press, Cambridge (1989)

Kei Sakai (Non-member) received B. E. from Department

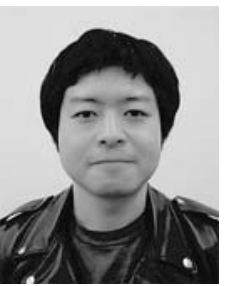
of Electrical and Electronic Engineering, Utsunomiya University, Tochigi, Japan in 2004. He has been interested in laser particle acceleration.

Kouichi Miyauchi (Non-member) received B. E. from De-

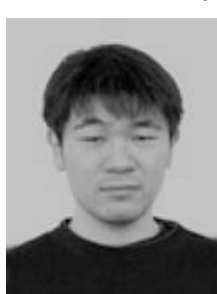
partment of Electrical and Electronic Engineering, Utsunomiya University, Tochigi, Japan in 2004. He has been interested in charged particle acceleration. 
Shuji Miyazaki (Non-member) received B. E. from Ad-

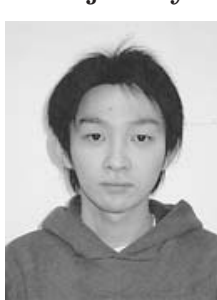
vanced Engineering Course, Gunma National College of Technology, Gunma, Japan in 2001 and he received a M. S. degree from Department of Electrical and Electronic Engineering, Utsunomiya University, Tochigi, Japan in 2004. He has been interested in laser particle acceleration and laser-matter interaction.

Qing Kong (Non-member) received Ph. D. from Fudan

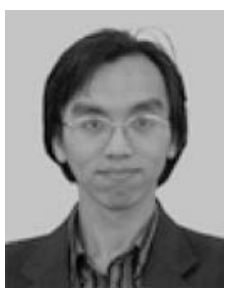
University, Shanghai, China in 2002. He is now an associate professor in Fudan University, China. He has been interested in particle laser acceleration and interaction between the matter and the intense laser.
Takashi Kikuchi (Non-member) received $\mathrm{Ph}$. D from

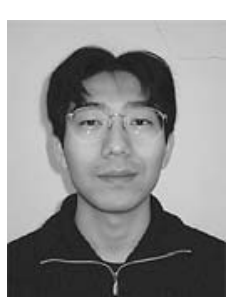
Tokyo Institute of Technology (TIT), Tokyo, Japan in 2003. In 2004 he became a research associate in Department of Electrical and Electronic Engineering, Utsunomiya University, Tochigi, Japan. He has been interested in heavy ion inertial fusion, spacecharge-dominated beam physics, and computational physics.

Shigeo Kawata (Member) received $\mathrm{Ph}$. D from Tokyo In-

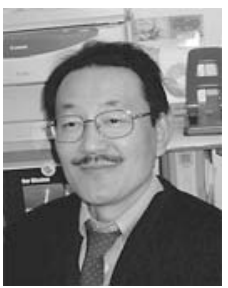
stitute of Technology(TIT), Tokyo, Japan in 1985. In 1981 he became a research associate in Dept. of Energy Sciences, TIT, in 1986 he became an associate professor in $\mathrm{Na}-$ gaoka Univ. of Tech. and in 1999 he became a professor in Utsunomiya University. $\mathrm{He}$ has been interested in inertial confinement fusion, energy problem, computational physics, and computer-assisted problem solving envi- 